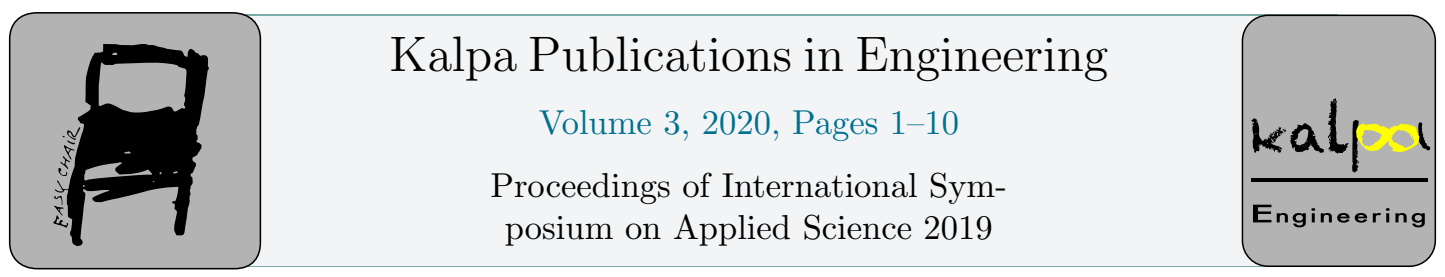

\title{
An Investigation into the Effects of Holes, Inclusion on Crack Growth in Composite Material under Dynamic Load by Extended Twice - Interpolation Finite Element Method
}

\author{
Kim Bang Tran ${ }^{1}$, Tich Thien Truong ${ }^{1}$, \\ ${ }^{1}$ VNUHCM - Ho Chi Minh City University of Technology \\ tkbang@hcmut.edu.vn
}

\begin{abstract}
The mixing of hard inclusions into materials is one way to strengthen and increase the durability of the materials, creating granular composite materials. However, apart from the hard inclusions in material, other impurities (soft inclusions) and holes still have the ability to appear and affect the durability of the material. The existence of holes, impurities will affect the behavior of the crack. In this paper, the authors will investigate the effects of holes, hard inclusions, soft inclusions on the behavior of crack when growing under the effect of dynamic loads by extended twice-interpolation finite element method-XTFEM. The results of dynamic stress intensity factor obtained from numerical examples will clearly show the impact of different types of particles on cracks.. The results obtained by XTFEM will be compared with the results published in a prestigious international scientific journal to verify reliability.

Keywords: crack, dynamic, extended, hole, hard inclusion, soft inclusion, twice interpolation, XTFEM
\end{abstract}

\section{Introduction}

The Extended Finite Element Method (XFEM) was proposed by Belytschko and Black in 1999 using the enrichment function to describe the physical properties of discontinuous boundaries, had certain advantages in analyzing mechanical behavior of materials with cracks, inclusions, holes and including composite materials.

Granular composite materials are widely applied in modern industry. Due to the unique properties of this material, many discontinuous objects will exist inside and impact their mechanical behavior. Therefore, XFEM is an effective tool for scientists to study the behavior of this material. One of the important issues is to study the effect of holes and inclusions on the behavior of cracks in composite 
materials. Yan and Park [1] applied XFEM to simulate the crack propagation near discontinuous boundary of the material. Singh et al. [2] studied the effect of the number of holes in an area domain on the change of stress intensity factor at the crack tip. Later, Singh et al. [3] also evaluated fatigue behavior of structures containing as holes, cracks and inclusions. S. Jiang et al. [4] also used XFEM to study the effect of holes on the dynamic behavior of cracks.

However, in order to increase the reliability in analyzing discontinuous problems with XFEM, the smoothness of stress and strain fields must be improved. Most recently, Bui et al. [5] succeeded in formulating consecutive-interpolation quadrilateral element, based on the idea in [6]. Stress fields become continuous and the reliability of computational results increase significantly. Therefore, Extended Twice-Interpolation Finite Element Method (XTFEM) was developed to take advantage of the XFEM and the twice interpolation idea.

As stated above, the analysis of the influence of inclusions and holes on cracks has been done quite a lot in previous studies. However, investigating the effect of holes and inclusions on the behavior of cracks as the crack grow, passing through the distribution of particles and holes under the effect of dynamic loads, has not been studied much. This paper applies XTFEM to crack, inclusions and holes to investigate the impact of them on the stress intensity factor under dynamic load over time. The set of problems include investigating the specific impact of holes when cracks grow; investigate the specific influence of inclusions when the crack grows and investigate the simultaneous impact of holes and inclusions on the crack. In particular, two types of hard and soft inclusions will be considered. The numerical results are compared with the published results of prestigious international scientific papers.

\section{Extended twice - interpolation finite element method for discontinuity problems}

\subsection{Twice - interpolation element shape functions for quadrilateral element}

Let $\mathbf{x}=(x, y)$ be a point in a quadrilateral element with node $i, j, k, m$. We here denote by $S_{i}, S_{j}, S_{k}$ and $S_{m}$ elements that share node $i, j, k$ and $m$, respectively. The supporting nodes for the point $\mathbf{x}$ in the twice - interpolation method involve all nodals of elements $S_{i}, S_{j}, S_{k}$ and $S_{m}$. According to [5], the trial solution at point $\mathbf{x}$ can be written as:

$$
\hat{u}(\mathbf{x})=\sum_{f=1}^{n_{s}} \hat{N}_{f}(\mathbf{x}) u_{f}
$$

In equation (1), the consecutive (or twice)-interpolation shape function $\hat{N}_{f}$ is determined by:

$$
\begin{aligned}
\hat{N}_{f}(\mathbf{x})= & \phi_{i} N_{f}^{[i]}+\phi_{\mathrm{ix}} \bar{N}_{f, x}^{[i]}+\phi_{i y} \bar{N}_{f, y}^{[i]}+\phi_{j} N_{f}^{[j]}+\phi_{j x} \bar{N}_{f, x}^{[j]}+\phi_{j y} \bar{N}_{f, y}^{[j]} \\
& +\phi_{k} N_{f}^{[k]}+\phi_{k x} \bar{N}_{f, x}^{[k]}+\phi_{k y} \bar{N}_{f, y}^{[k]}+\phi_{m} N_{f}^{[m]}+\phi_{m x} \bar{N}_{f, x}^{[m]}+\phi_{m y} \bar{N}_{f, y}^{[m]}
\end{aligned}
$$

Where $u_{f}$ denotes the nodal displacement vector at node $f, N_{f}^{[i]}$ is the shape function of node $f$

with value at $\mathbf{x}_{i}, \bar{N}_{f, x}^{[i]}$ is the average derivative of the shape functions of $N_{f}^{[i]}$ with respect to $x$ and $n_{s}$ is the total number of nodes supporting points $\mathbf{x}$. According to [5] and [6], the average derivative of the shape functions at node $i$ is given as follows: 


$$
\bar{N}_{f, x}^{[i]}=\sum_{e \in S_{i}}\left(\omega_{e} N_{f, x}^{[i][e]}\right), \bar{N}_{f, y}^{[i]}=\sum_{e \in S_{i}}\left(\omega_{e} N_{f, y}^{[i][e]}\right), \omega_{e}=\frac{\Delta_{e}}{\sum_{e^{\prime} \in S_{i}} \Delta_{e^{\prime}}}
$$

According to the equation (3), $N_{f, x}^{[i][e]}$ is the derivative of $N_{f}^{[i]}$ with respect to $x$ in element $e, \omega_{e}$ is the weight function of the element $e$ in the element domain $S_{i}, \Delta_{\mathrm{e}}$ and $\Delta_{\mathrm{e}}$, is the area of the element $e$ and $e^{\prime}$.

In equation (2), the functions $\phi_{i}, \phi_{i x}, \phi_{i y}$ forming the polynomial basis associated with nodal $i$ must satisfy the following conditions:

$$
\begin{aligned}
& \phi_{i}\left(\mathbf{x}_{l}\right)=\delta_{i l}, \quad \phi_{i, x}\left(\mathbf{x}_{l}\right)=0, \quad \phi_{i, y}\left(\mathbf{x}_{l}\right)=0 \\
& \begin{array}{l}
\phi_{i x}\left(\mathbf{x}_{l}\right)=0, \phi_{i x, x}\left(\mathbf{x}_{l}\right)=\delta_{i l}, \phi_{i x, y}\left(\mathbf{x}_{l}\right)=0 \\
\phi_{i, y}\left(\mathbf{x}_{l}\right)=0, \phi_{i, y}\left(\mathbf{x}_{l}\right)=0, \quad \phi_{i y, y}\left(\mathbf{x}_{l}\right)=\delta_{i l}
\end{array}, \delta_{i l}=\left\{\begin{array}{l}
1, \text { with } i=l \\
0, \text { with } i \neq l
\end{array}\right.
\end{aligned}
$$

where $l$ is any one of the indices $i, j, k$, and $m$

These polynomial basis functions $\phi_{i}, \phi_{i x}, \phi_{i y}$ for the twice - interpolation element are given by:

$$
\begin{aligned}
\phi_{i}= & L_{i}+L_{i}^{2} L_{j}+L_{i}^{2} L_{k}+L_{i}^{2} L_{m}-L_{i} L_{j}^{2}-L_{i} L_{k}^{2}-L_{i} L_{m}^{2} \\
\phi_{i x}= & -\left(x_{i}-x_{j}\right)\left(L_{i}^{2} L_{j}+b L_{i} L_{j} L_{k}+b L_{i} L_{j} L_{m}\right) \\
& -\left(x_{i}-x_{k}\right)\left(L_{i}^{2} L_{k}+b L_{i} L_{k} L_{m}+b L_{i} L_{k} L_{j}\right) \\
& -\left(x_{i}-x_{m}\right)\left(L_{i}^{2} L_{m}+b L_{i} L_{m} L_{j}+b L_{i} L_{m} L_{k}\right)
\end{aligned}
$$

$\phi_{\text {iy }}$ is calculated similarly by replacing variable $\mathrm{x}$ with the variable $\mathrm{y}$.

With $b=1 / 2, L_{i}, L_{j}, L_{k}$ and $L_{m}$ are shape function of the point of interest $\mathbf{x}$ in the quadrilateral element $i, j, k, m$ in basis finite element method. The functions $\phi_{j}, \phi_{j x}, \phi_{j y}, \phi_{k}, \phi_{k x}, \phi_{k y}$ and $\phi_{m}, \phi_{m x}, \phi_{m y}$ can be also calculated in the same manner by a circulatory permutation of indices $i, j, k$, and $m$.

\subsection{XTFEM approximations for dynamic crack problem with inclusion and hole}

Consider $\mathbf{x}$, a point in a finite element model. Also assume there is some discontinuities including cracks, holes and hard inclusions coexist. A result the XTFEM approximation of the displacements for crack, inclusion and hole can be written as:

$$
\hat{u}^{h}(\mathbf{x})=\hat{u}(\mathbf{x})+\hat{u}^{e n r}(\mathbf{x})=\sum_{f=1}^{n_{s}} \hat{N}_{f}(\mathbf{x}) u_{f}+\sum_{l=1}^{n p} \sum_{k=1}^{m} \hat{N}_{k}(\mathbf{x}) \hat{\psi}^{l}(\mathbf{x}) a_{k}^{l}
$$

Where $n_{s}$ is the number of nodes supporting points with $\mathrm{x}$ coordinates; $n p$ is the number of discontinuous conditions that occur with the element containing the point $\mathrm{x}$; for the element containing crack tip, $n p=4$, and for the element cut by discontinuous boundary of crack edge, hole, and inclusion, $n p=1 ; m$ is the number of nodes enriched in each $n p ; \hat{\psi}$ is the enrichment function in each discontinuous condition (crack, hole, inclusion) $n p ; a$ is the degrees of freedom corresponding to the enriched node.

Ignore the damping phenomenon, $\hat{u}$ is the real degrees of freedom, $\hat{u}^{\text {spl }}, \hat{u}^{\text {tip }}, \hat{u}^{\text {hole }}, \hat{u}^{\text {inc }}$ are virtual degrees of freedom of discontinuities including crack edge, crack tip, hole and inclusion calculated based on twice interpolation. Enrichment stiffness matrix $\mathbf{K}$ and enrichment mass matrix $\mathbf{M}$ will have the general form as follows: 
An Investigation into the Effects of Holes, Inclusion on Crack Growth... K.B. Tran and T.T. Truong

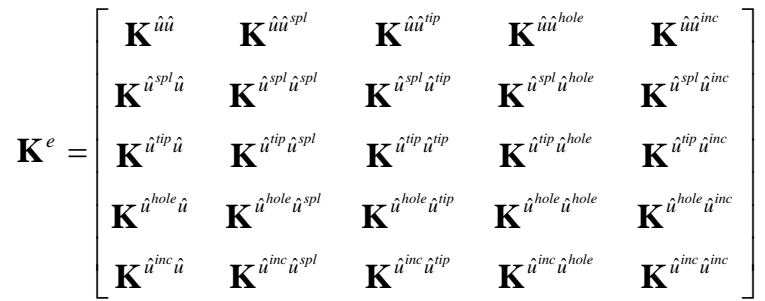

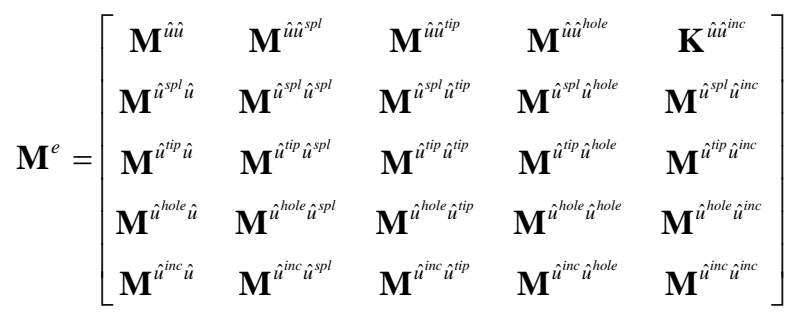

Enrichment matrix $\mathbf{B}$ and $\mathbf{N}$ for discontinuous cases including crack edge, crack tip, hole and inclusion will be as follows:

$$
\begin{aligned}
\mathbf{B}^{e} & =\left[\begin{array}{lllll}
\mathbf{B}^{\text {TFEM }} & \mathbf{B}^{\text {spl }} & \mathbf{B}^{\text {tip }} & \mathbf{B}^{\text {hole }} & \mathbf{B}^{\text {inc }}
\end{array}\right] \\
\mathbf{N}^{e} & =\left[\begin{array}{lllll}
\mathbf{N}^{\text {TFEM }} & \mathbf{N}^{\text {spl }} & \mathbf{N}^{\text {tip }} & \mathbf{N}^{\text {hole }} & \mathbf{N}^{\text {inc }}
\end{array}\right]
\end{aligned}
$$

In the case of crack edge:

$$
\begin{aligned}
\mathbf{B}_{i}^{s p l} & =\left[\begin{array}{cc}
\left(\hat{N}_{i}\left[H(\xi)-H\left(\xi_{i}\right)\right]\right)_{, x} & 0 \\
0 & \left(\hat{N}_{i}\left[H(\xi)-H\left(\xi_{i}\right)\right]\right)_{, y} \\
\left(\hat{N}_{i}\left[H(\xi)-H\left(\xi_{i}\right)\right]\right)_{, y} & \left(\hat{N}_{i}\left[H(\xi)-H\left(\xi_{i}\right)\right]\right)_{, x}
\end{array}\right] \\
\mathbf{N}_{i}^{s p l} & =\left[\begin{array}{cc}
\hat{N}_{i}\left[H(\xi)-H\left(\xi_{i}\right)\right] & 0 \\
0 & \hat{N}_{i}\left[H(\xi)-H\left(\xi_{i}\right)\right]
\end{array}\right] \\
H(\mathbf{x}) & =\left\{\begin{array}{cc}
1 & \phi(\mathbf{x})>0 \\
-1 & \phi(\mathbf{x})<0
\end{array}\right.
\end{aligned}
$$

In the case of hole:

$$
\begin{aligned}
\mathbf{B}_{i}^{\text {hole }} & =\left[\begin{array}{cc}
\hat{N}_{i, x}\left[V(\xi)-V\left(\xi_{i}\right)\right] & 0 \\
0 & \hat{N}_{i, y}\left[V(\xi)-V\left(\xi_{i}\right)\right] \\
\hat{N}_{i, y}\left[V(\xi)-V\left(\xi_{i}\right)\right] & \hat{N}_{i, x}\left[V(\xi)-V\left(\xi_{i}\right)\right]
\end{array}\right] \\
\mathbf{N}_{i}^{\text {hole }} & =\left[\begin{array}{cc}
\hat{N}_{i}\left[V(\xi)-V\left(\xi_{i}\right)\right] & 0 \\
0 & \hat{N}_{i}\left[V(\xi)-V\left(\xi_{i}\right)\right]
\end{array}\right] \\
V(\mathbf{x}) & = \begin{cases}1 & \phi(\mathbf{x})>0 \\
0 & \phi(\mathbf{x})<0\end{cases}
\end{aligned}
$$

In the case of inclusion: 
An Investigation into the Effects of Holes, Inclusion on Crack Growth... K.B. Tran and T.T. Truong

$$
\begin{aligned}
& \mathbf{B}_{i}^{i n c}=\left[\begin{array}{cc}
\hat{N}_{i, x} \chi(\xi)+\hat{N}_{i} \chi(\xi)_{, x} & 0 \\
0 & \hat{N}_{i, y} \chi(\xi)+\hat{N}_{i} \chi(\xi)_{, y} \\
\hat{N}_{i, y} \chi(\xi)+\hat{N}_{i} \chi(\xi)_{, y} & \hat{N}_{i, x} \chi(\xi)+\hat{N}_{i} \chi(\xi)_{, x}
\end{array}\right] \\
& \mathbf{N}_{i}^{i n c}=\left[\begin{array}{cc}
\hat{N}_{i} \chi(\xi) & 0 \\
0 & \hat{N}_{i} \chi(\xi)
\end{array}\right] \\
& \chi(\mathbf{x})=\sum_{i} \hat{N}_{i}(\mathbf{x})\left|\phi_{i}\right|-\left|\sum_{i} \hat{N}_{i}(\mathbf{x}) \phi_{i}\right|
\end{aligned}
$$

$\hat{N}_{i}$ is the twice-interpolation shape function at enriched node $i$.

$H(\xi)$ is the Heaviside function at Gauss point.

$H\left(\xi_{i}\right)$ is the Heaviside function at node $\mathrm{i}$.

$\phi_{i}$ is the signed distance function at node $\mathrm{i}$ of an enriched element.

In general, the geometry of a discontinuous boundary can be characterized by a zero value of level set function $\phi(\mathbf{x}, t)=0$ and $\phi(\mathbf{x}, t)$ is determined as follows

$$
\phi(\mathbf{x}, t)= \pm \min _{\mathbf{x}_{\Gamma} \in \Gamma(t)}\left\|\mathbf{x}-\mathbf{x}_{\Gamma}\right\|
$$

With $\Gamma$ is discontinuous boundary, $t$ is a set of plus and minus signs defined by a sign of $\mathbf{x}-\mathbf{x}_{\Gamma}$ In the case of crack tip:

$$
\begin{aligned}
& \mathbf{B}_{i}^{t i p \alpha}=\left[\begin{array}{cc}
\left(\hat{N}_{i}\left[F_{\alpha}-F_{\alpha i}\right]\right)_{, x} & 0 \\
0 & \left(\hat{N}_{i}\left[F_{\alpha}-F_{\alpha i}\right]\right)_{, y} \\
\left(\hat{N}_{i}\left[F_{\alpha}-F_{\alpha i}\right]\right)_{, y} & \left(\hat{N}_{i}\left[F_{\alpha}-F_{\alpha i}\right]\right)_{, x}
\end{array}\right] \alpha=1,2,3,4 \\
& \mathbf{N}_{i}^{t i p \alpha}=\left[\begin{array}{cc}
\hat{N}_{i}\left[F_{\alpha}-F_{\alpha i}\right] & 0 \\
0 & \hat{N}_{i}\left[F_{\alpha}-F_{\alpha i}\right]
\end{array}\right] \alpha=1,2,3,4 \\
&\left\{F_{\alpha}(r, \theta)\right\}_{\alpha=1}^{4}=\left\{\sqrt{r} \sin \left(\frac{\theta}{2}\right), \sqrt{r} \cos \left(\frac{\theta}{2}\right), \sqrt{r} \sin \left(\frac{\theta}{2}\right) \sin (\theta), \sqrt{r} \cos \left(\frac{\theta}{2}\right) \sin (\theta)\right\}
\end{aligned}
$$

Where $(r, \theta)$ are components of the local polar coordinate system located at crack tip.

\subsection{Interaction integral for dynamic crack problem}

The J-integration method for XTFEM uses twice interpolation displacement field, with a larger number of nodes and support domains than XFEM. The analytical form of integral $\mathbf{J}$ for the dynamic crack problem is given as follows

$$
J_{K}=\int_{\Gamma+\Gamma_{C}}\left((W+K) n_{k}-t_{i} \frac{\partial \hat{u}_{j}}{\partial x_{k}}\right) d \Gamma+\int_{V_{\Gamma}-V_{e}}\left(\left(\rho \ddot{\hat{u}}_{i}-f_{i}\right) \frac{\partial \hat{u}_{i}}{\partial x_{k}}-\rho \frac{\partial \dot{\hat{u}}_{i}}{\partial x_{k}} \dot{\hat{u}}_{i}\right) d A
$$

With $u_{i}, t_{i}, f_{i}, n_{k}$ and $\rho$ are denoted for displacement, pressure, volume force, normal vector and density. $\mathrm{W}$ is the strain energy density and $\mathrm{K}$ is the kinetic energy density. An alternative form of interaction integral proposed to make it easy to apply to finite element methods is given as follows: 


$$
J_{K}^{\prime}=\int_{V_{\Gamma}}\left(\sigma_{i j} \frac{\partial \hat{u}_{j}}{\partial x_{k}}-(W+K)\right) q_{, k} d A+\int_{V_{\Gamma}}\left(\left(\rho \ddot{\hat{u}}_{i}-f_{i}\right) \frac{\partial \hat{u}_{i}}{\partial x_{k}}-\rho \frac{\partial \dot{\hat{u}}_{i}}{\partial x_{k}} \dot{u}_{i}\right) q d A
$$

Where A is the area domain surrounded by boundary $\Gamma, q$ is a smooth function which value varies from $q=1$ near the crack tip to $q=0$ at the position outside the boundary $\Gamma$. In this case, $\mathrm{q}$ is assumed to vary linearly from 1 to 0 .

\section{Numerical examples}

\subsection{Centre-cracked plate with one circular inclusion}

In the first problem, the influence of inclusion on stress intensity factor will be investigated. The plate has dimensions $\mathrm{W}=20 \mathrm{~mm}, \mathrm{H}=40 \mathrm{~mm}$, the crack is in the middle of the plate with a $=2.4 \mathrm{~mm}$. The uniformly distributed force $\mathrm{f}_{0}=1 \mathrm{~N} / \mathrm{m}$ on the top and bottom edges of the plate, acting from 0 to $13.62 \mu \mathrm{s}$ (Figure 1c). Time step $\Delta \mathrm{t}=0.05 \mu \mathrm{s}$. The matrix material has elastic modulus $\mathrm{E}=199.992 \mathrm{GPa}$, Poisson coefficient $v=0.3$, density $\rho=5000 \mathrm{~kg} / \mathrm{m}^{3}$. The inclusion have radius $\mathrm{r}=2 \mathrm{~mm}$, located with a distance $\mathrm{d}=6 \mathrm{~mm}$. Two cases are considered: hard inclusion with elastic modulus $\mathrm{E}=199.992 \times 10^{3} \mathrm{GPa}$ (Figure 1a) and soft inclusion with elastic modulus $\mathrm{E}=199.992 \mathrm{GPa}$ (Figure 1b). The Poisson coefficient of hard and soft inclusion are both equal to 0.3 . The density of hard and soft inclusion are both equal to $5000 \mathrm{~kg} / \mathrm{m}^{3}$. Plane strain state is investigated. The propagation velocity of vertical wave $C_{d}=7.34 \mathrm{~mm} \mu \mathrm{s}-1$. The results obtained from XTFEM are compared with results published from [4]. Time will be normalized as follows:

$t_{\text {normalzied }}=2 C_{d} t / h \quad$ where $\mathrm{h}=\mathrm{H}$ is the plate height

Mode I dynamic stress intensity factor will be normalized as follows:

$$
K_{I}^{*}=\frac{K_{I}}{K_{0}} ; \quad K_{0}=f_{0} \sqrt{\pi a}
$$

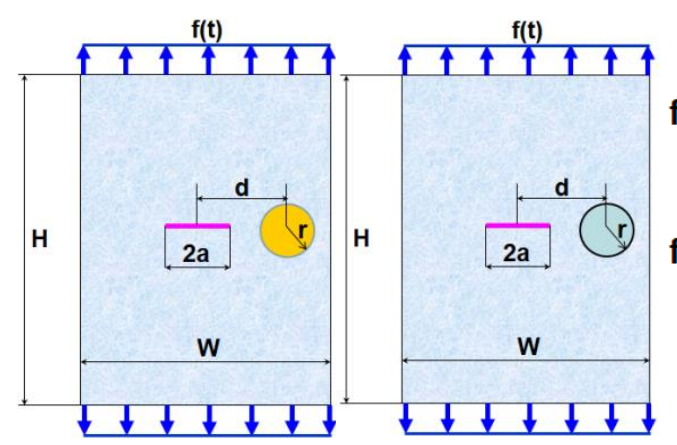

(a)

(b)

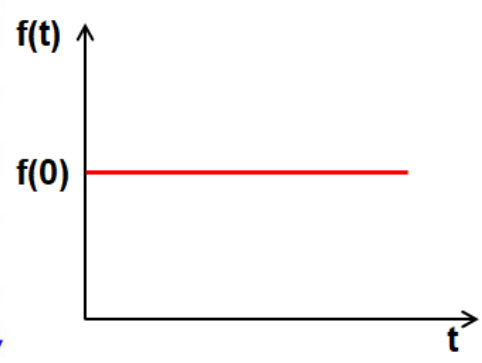

(c)

Figure 1: Plate with crack near inclusion with: (a) hard inclusion and (b) soft inclusion

The results of dynamic stress intensity factor for the two cases of hard and soft inclusion obtained from XTFEM are quite similar to the XFEM results referenced from [4]. It can be seen that in the case of hard inclusion (Figure 2a) near the crack tip, the dynamic stress intensity factor is significantly reduced compared to the case of soft inclusion (Figure $2 b$ ) near the crack tip. The appearance of hard inclusion near the crack tip will reduce the concentration of stress at the crack tip, limiting damage and reinforcing the material more firmly. 


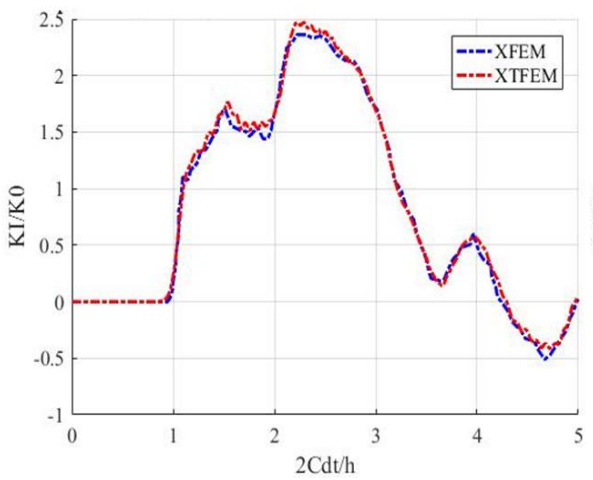

(a)

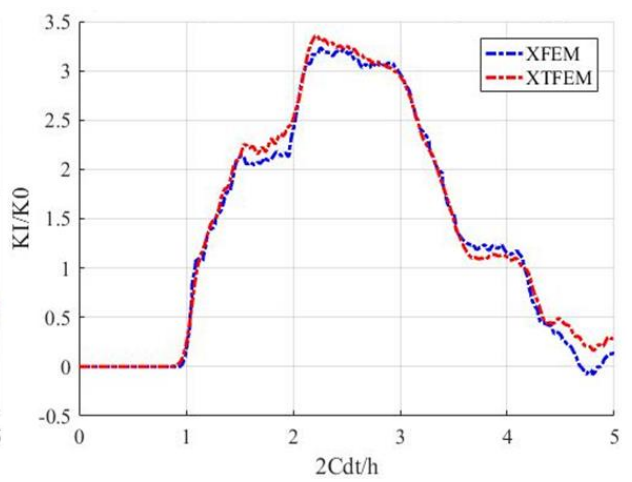

(b)

Figure 2: Dynamic stress intensity factor under the influence of (a) hard inclusion and (b) soft inclusion

\subsection{Edge-cracked plate with two circular inclusions}

In the next example, the influence of holes, soft inclusions, hard inclusions on dynamic stress intensity factor in case of crack growth will be investigated. Considering the cracked plate containing holes (figure 3a), soft inclusions (Figure 3b) and hard inclusions, respectively (Figure 3c). The plate has dimensions of $\mathrm{W}=5 \mathrm{~m}, \mathrm{H}=5 \mathrm{~m}$, contains an edge crack with initial size $\mathrm{a}=0.6 \mathrm{~m}$. The holes and inclusions are the same size with $\mathrm{r}=0.6 \mathrm{~m}$. Inclusions are located at $\mathrm{O}_{1}(2.50,1.25) \mathrm{m}$ and $\mathrm{O}_{2}(2.5,3.75) \mathrm{m}$. The matrix material has elastic modulus $\mathrm{E}=3 \times 10^{7} \mathrm{~Pa}$, Poisson's coefficient $v=0.3$, density $\rho=7000 \mathrm{~kg} / \mathrm{m}^{3}$. Hard inclusion have an elastic modulus $\mathrm{E}=3 \times 10^{9} \mathrm{~Pa}$ and soft inclusion have an elastic modulus $\mathrm{E}=3 \times 10^{6} \mathrm{~Pa}$. The poisson coefficient $v$ of both hard and soft inclusion is equal to 0.3 . The density of hard and soft inclusion is equal to $6000 \mathrm{~kg} / \mathrm{m}^{3}$. The load over time is described as Figure $6 \mathrm{~b}$. The bottom edge of the plate is clamped. Plane strain state is investigated. The propagation

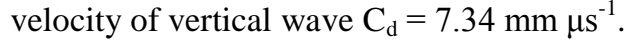

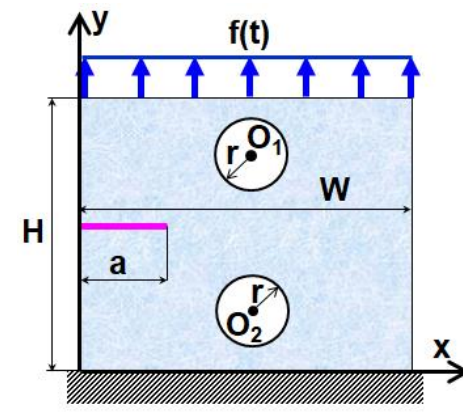

(a)

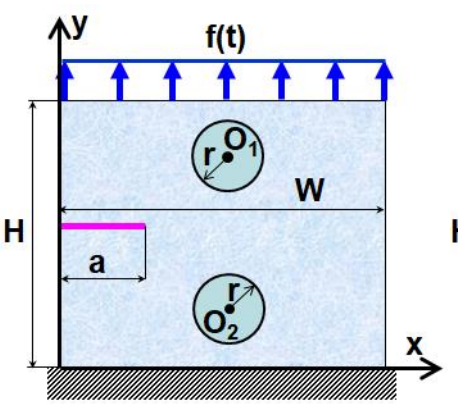

(b)

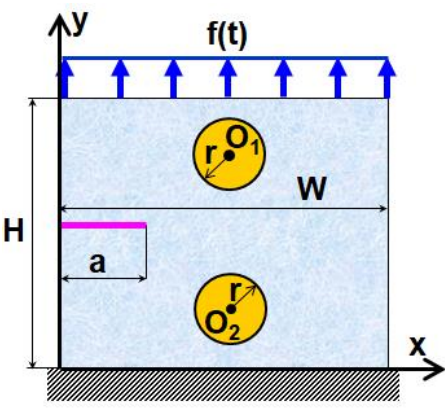

(c)

Figure 3: Plate with crack near inclusion with: (a) holes; (b) soft inclusion and (c) hard inclusion

Stress field results in Figure 4 show that when the crack tip moves near the inclusion, with two cases of holes and soft inclusion, the stress is most concentrated mainly at the crack tip (Figure 4a and $4 \mathrm{~b}$ ). In the case of hard inclusion, the stress is still concentrated in the grain boundary near the crack (Figure 4c). 


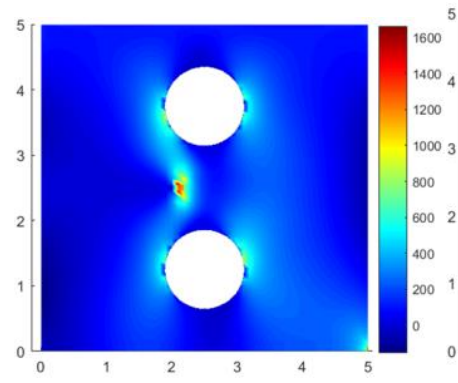

(a)

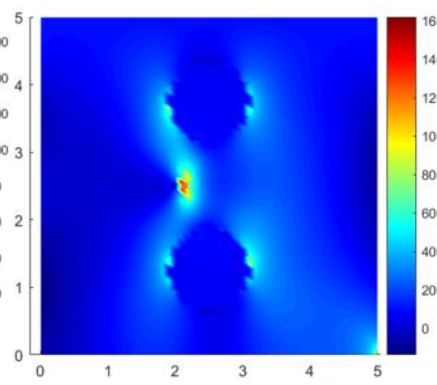

(b)

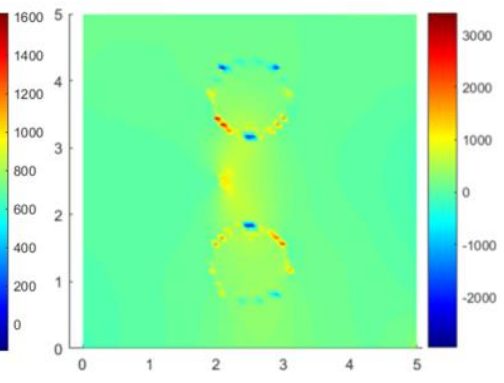

(c)

Figure 4: Stress field $\sigma_{\mathrm{yy}}$ at the time of $0.3 \mathrm{~s}$ distributed in the cracked plate together with:

(a) holes; (b) soft inclusion s and (c) hard inclusion

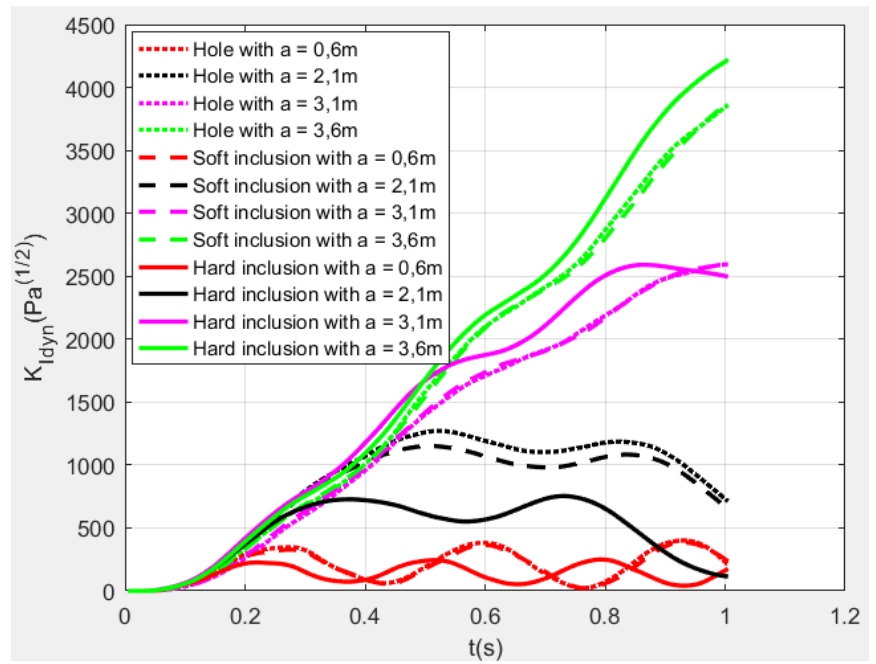

Figure 5: The variation of dynamic stress intensity factor according to crack size with cases of cracked plate containing holes, soft inclusions and hard inclusions, respectively

Figure 5 depicts the variation of dynamic stress intensity factor when cracked plate contain holes, hard and soft inclusions, respectively, with 4 different crack sizes. It can be seen that when the crack tip approached toward the hard particle region $(\mathrm{a}=0.6 \mathrm{~m}$ to $\mathrm{a}=2.1 \mathrm{~m})$, the dynamic strength factor was significantly smaller than in the case of holes and soft inclusions. This is similar to the results obtained from problem 1 . As the crack tip gets farther away from the hard inclusion domain $(\mathrm{a}=3.1 \mathrm{~m}$ to $\mathrm{a}=3.6 \mathrm{~m})$, the dynamic stress intensity factor tends to increase, over compared to the other two cases. Thus it can be seen that when the material is mixed with hard particles, these particles have the ability to prevent the development of cracks when the crack tip enters the domain containing the hard particles.

\subsection{Edge-cracked plate with two circular holes and inclusions}

In the last example, the simultaneous effects of hard inclusions and holes on crack growth will be investigated. Consider the cracked plate that contain holes and hard inclusions simultaneously. The dimensions and material properties are given in the same way as the previous problem. The load over 
time is described as Figure $6 \mathrm{~b}$. The plate contains 2 holes and 2 hard inclusions with the coordinates of the centers of the particles respectively are: $\mathrm{O}_{1}(1.25,1.25) \mathrm{m}, \mathrm{O}_{2}(1.25,3.75) \mathrm{m}, \mathrm{O}_{3}(3.75,1.25) \mathrm{m}$, $\mathrm{O}_{4}(3.75,3.75) \mathrm{m}$. Hard inclusions and holes have equal size of $0.6 \mathrm{~m}$. The initial crack length is $0.6 \mathrm{~m}$.

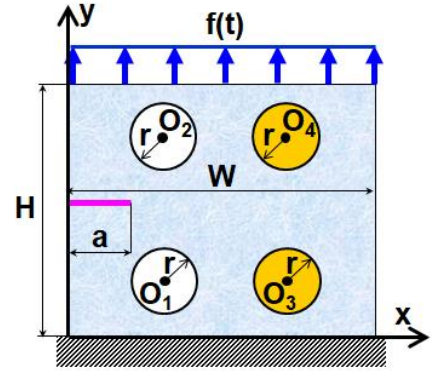

(a)

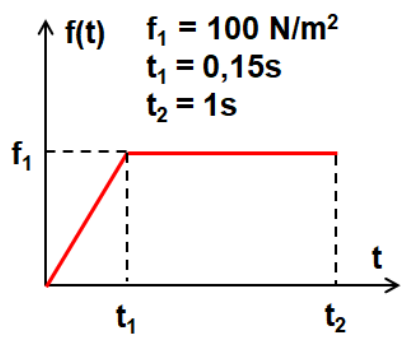

(b)

Figure 6: Plate containing cracks, holes, and hard inclusions and subjected the load over time

The the variation of dynamic stress intensity factor according to crack size is shown in Figure 7.

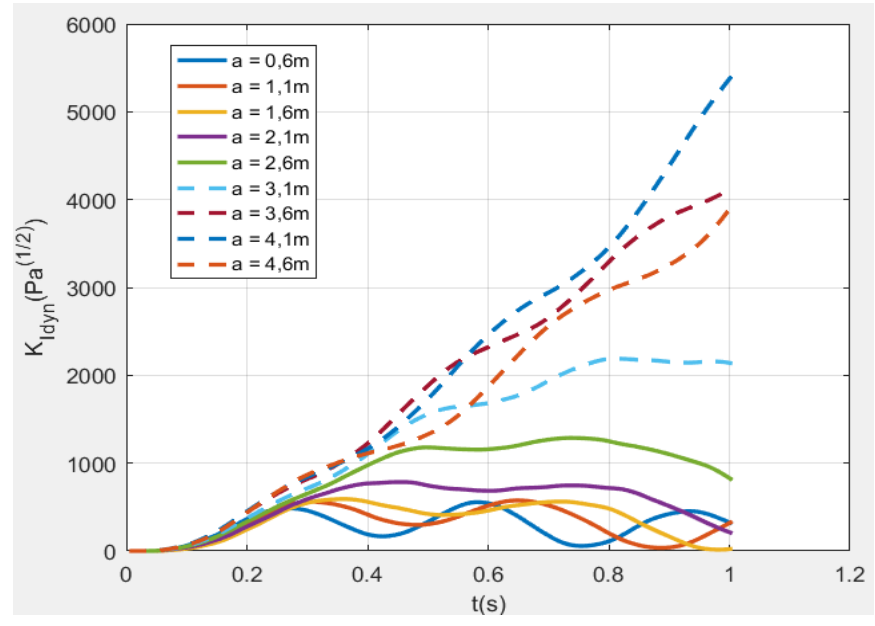

Figure 7: The variation of dynamic stress intensity factor according to crack size with cases of cracked plate containing both holes and hard inclusions simultaneously

It can be seen that over a period of 1 second, the dynamic stress intensity factor tends to increase and the oscillation decreases as the crack size increases. However, there is a phenomenon where the dynamic stress intensity factor is reduced when the crack size is equal to $4.6 \mathrm{~m}$, and the crack tip is nearly touching the right edge of the sheet.

Figure 8 shows that as the crack changes in size, the stress is increasingly concentrated in the hard inclusion above and below the crack, significantly affecting the stress intensity factor at the crack tip. 

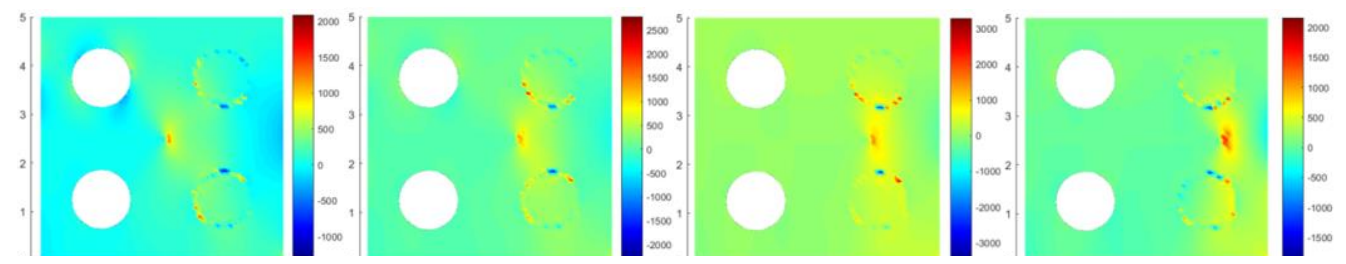

Figure 8: Stress field $\sigma_{y y}$ at time $0.3 \mathrm{~s}$ with length of the crack, respectively $\mathrm{a}=3.1 \mathrm{~m}, \mathrm{a}=3.6 \mathrm{~m}$, $\mathrm{a}=4.1 \mathrm{~m}$ and $\mathrm{a}=4.6 \mathrm{~m}$

\section{Conclusions}

In this paper, the effect of holes and inclusions on cracks when the size of cracks changes has been investigated by extended twice interpolation finite element method (XTFEM) based on programming language Matlab. The results of analyzing the influence of soft and hard inclusions on cracks obtained from XTFEM are quite similar to those from reputable literature [4]. Some numerical examples were performed and analyzed the effects of holes, hard inclusions and soft inclusions on the crack when distributed near the crack when the crack size changed. Through the numerical results, it can be seen that the existence of hard inclusions before the crack tip has the ability to reduce the dynamic stress intensity factor of the crack and this has the ability to prevent the damage of cracked area.

\section{Acknowledgement}

This research is funded by Ho Chi Minh City University of Technology - VNU-HCM, under grant number T-KHUD-2018-22.

\section{References}

[1] Yan, Y. and Park, S. (2008), “An extended finite element method for modeling near-interfacial crack propagation in a layered structure", Int. J. Solid. Struct., 45, pp. 4756-4765.

[2] Singh, I.V., Mishra, B.K. and Bhattacharya, S. (2011), "XFEM simulation of cracks, holes and inclusions in functionally graded materials", Int. J. Mech. Mater. Des., 7, pp. 199-218.

[3] Singh, I.V., Mishra, B.K., Bhattacharya, S. and Patil, R.U. (2012), "The numerical simulation of fatigue crack growth using extended finite element method", Int. J. Fatigue, 36, pp. 109-119.

[4] S. Jiang, C. Du, C. Gu and X. Chen (2014), "XFEM analysis of the effects of voids, inclusions and other cracks on the dynamic stress intensity factor of a major crack", Fatigue Fract. Eng. Mater. Struct., 37, pp.1-17.

[5] Q.T. Bui, Q.D. Vo, Ch. Zhang, D.D. Nguyen (2014), "A consecutive-interpolation quadrilateral element (CQ4)", Formulation and Applications Finite Elem. Anal. Des., 84, pp.14-31.

[6] C. Zheng, S.C. Wu, X.H. Tang, J.H. Zhang (2010), “A novel twice-interpolation finite element method for solid mechanics problems", Acta Mech. Sin., 26, pp.265-278.

[7] S. Mohammadi (2012), XFEM fracture analysis of composites, John Wiley \& Sons. 